\title{
Nonnegative curvature on disk bundles
}

\author{
Lorenz J. Schwachhöfer
}

Preprint 2009-16

Dezember 2009

Fakultät für Mathematik

Technische Universität Dortmund

Vogelpothsweg 87

44227 Dortmund

$\underline{\text { tu-dortmund.de/MathPreprints }}$ 



\title{
Nonnegative curvature on disk bundles
}

\author{
Lorenz J. Schwachhöfer*
}

\section{Introduction}

The search for manifolds of nonnegative curvature ${ }^{1}$ is one of the classical problems in Riemannian geometry. While general obstructions are scarce, there are relatively few general classes of examples and construction methods. Hence, it is unclear how large one should expect the class of closed manifolds admitting a nonnegatively curved metric to be. For a survey of known examples, see e.g. [Z].

Apart from taking products, there are only two general methods to construct new nonnegatively curved metrics out of given spaces. One is the use of Riemannian submersions which non-decrease curvature by O'Neill's formula. The other is the glueing of two manifolds (which we call halves) along their common boundary. Typically, the boundary of each half is assumed to be totally geodesic or, slightly more restrictive, a collar metric. This in turn implies by the Soul theorem ([CG]) that each half is the total space of a disk bundle over a totally geodesic closed submanifold. In addition, the glueing map of the two boundaries must be an isometry.

While many examples can be constructed by such a glueing, its application is still limited. On the one hand, there is not too much known on the question which disk bundles over a nonnegatively curved compact manifold admit collar metrics of nonnegative curvature, and on the other hand, even if such metrics exist, the metric on the boundary is not arbitrary. Thus, glueing together two such disk bundles to a nonnegatively curved closed manifold is possible in special situations only.

For instance, if the disk bundle is homogeneous, then there always exist invariant nonnegatively curved collar metrics. However, the metric on the boundary of such a collar metric is restricted due to the existence of certain parallel Killing fields by a result of Perelman $([\mathrm{P}])$.

In this article, we will give a survey of known examples and describe some recent results which illustrate the difficulty in finding metrics on disk bundles which are suitable for this glueing construction.

\footnotetext{
${ }^{*}$ Supported by the Schwerpunktprogramm Differentialgeometrie of the Deutsche Forschungsgemeinschaft

${ }^{1}$ Throughout this article, the term "curvature" refers to the sectional curvature.
} 


\section{Normal homogeneous metrics and Cheeger deforma- tions}

Let $G$ be a compact Lie group with Lie algebra $\mathfrak{g}$, and let $g_{0}$ be a biinvariant metric on $G$, i.e., a metric for which all left and right translations are isometries. It is well known that each compact Lie group carries such a metric, and its curvature is given by

$$
\sec _{g_{0}}(x, y)=\frac{1}{4}|[x, y]|_{g_{0}}^{2} \geq 0
$$

for each orthonormal pair $x, y \in T_{g} G=d L_{g} \mathfrak{g} \cong \mathfrak{g}$. Let $H \subset G$ be a closed subgroup, and consider the compact homogeneous space $M:=G / H$. Then there is a unique $G$ invariant metric on $G / H$ which by abuse of notation we also denote by $g_{0}$, for which the canonical projection $G \rightarrow G / H$ becomes a Riemannian submersion. This metric on $G / H$ is called a normal homogeneous metric. Evidently, a normal homogeneous metric always has nonnegative curvature by O'Neill's formula.

Let $(M, g)$ be a Riemannian manifold, and suppose that $G$ acts isometrically on $M$. The action map

$$
G \times M \longrightarrow M
$$

may be regarded as a principal $G$-bundle with the free action of $G$ on $G \times M$ given by

$$
k \star(g, p):=\left(g k^{-1}, k \cdot p\right)
$$

for $g, k \in G$ and $p \in M$. Evidently, this action is isometric w.r.t. the product of a biinvariant metric on $G$ and the given metric on $M$.

Definition 2.1 Let $(M, g)$ be a Riemannian manifold, and let $G$ be a Lie group with a biinvariant metric $g_{0}$ which acts isometrically on $M$. Then for $\lambda>0$, the metric $g_{\lambda}$ on $M$ for which the action map

$$
\left(G \times M, \lambda g_{0} \oplus g\right) \longrightarrow\left(M, g_{\lambda}\right)
$$

becomes a Riemannian submersion is called a Cheeger deformation of $g$.

Since Cheeger deformations are Riemannian submersions and hence curvature non-decreasing by O'Neills formula, it follows that $g_{\lambda}$ has nonnegative curvature whenever $g$ does. Moreover, $g_{\lambda}$ may have nonnegative curvature even if $g$ has some negative curvature.

Proposition 2.2 Let $g$ be a $G$-invariant metric on $M$ and let $g_{\lambda}$ denote the Cheeger deformation of $g$ for $\lambda>0$. Then

1. $\lim _{\lambda \rightarrow \infty} g_{\lambda}=g$.

2. $\left(g_{\lambda}\right)_{\mu}=g_{\frac{\lambda \mu}{\lambda+\mu}}$. That is, the Cheeger deformation of a Cheeger deformation is again a Cheeger deformation. 
3. If $g_{\lambda_{0}}$ has nonnegative curvature for some $\lambda_{0}>0$, then so does $g_{\lambda}$ for any $\lambda<\lambda_{0}$.

4. If $M=G / H$ is a homogeneous space with a $G$-invariant metric $g$, then $\lim _{\lambda \rightarrow 0} \frac{1}{\lambda} g_{\lambda}=$ $g_{0}$, where $g_{0}$ is the normal homogeneous metric on $G / H$ induced by the biinvariant metric $g_{0}$ on $G$.

Proof. Let $p \in M$, and let $N:=G \cdot p \subset M$ be the $G$-orbit through $p$. We decompose the tangent space $g$-orthogonally as

$$
T_{p} M=T_{p} N \oplus S_{p} .
$$

We write $N=G / H$ with $H:=\operatorname{Stab}(p) \subset G$, and choose the orthogonal decomposition of the Lie algebra $\mathfrak{g}=\mathfrak{h} \oplus \mathfrak{m}$, so that $\mathfrak{m} \cong T_{p} N$ with the identification $x \mapsto\left(x^{*}\right)_{p}$, where $x^{*}$ denotes the action field of $x \in \mathfrak{g}$. Let $\varphi: \mathfrak{m} \rightarrow \mathfrak{m}$ be the self-adjoint map for which

$$
g\left(x^{*}, y^{*}\right)_{p}=g_{0}(\varphi(x), y),
$$

where $g_{0}$ is the given biinvariant inner product on $\mathfrak{g}$. The tangent space of the fiber of the submersion $G \times M \rightarrow M$ at $(e, p)$ is given by $\left\{\left(-x,\left(x^{*}\right)_{p}\right) \mid x \in \mathfrak{g}\right\}$, hence its orthogonal complement w.r.t. $\left(\lambda g_{0}\right) \oplus g$ is

$$
\mathcal{H}_{(e, p)}=\left\{\left(\varphi(x), \lambda\left(x^{*}\right)_{p}\right) \mid x \in \mathfrak{m}\right\} \oplus S_{p} .
$$

Therefore, the horizontal lift of $\left(x^{*}\right)_{p}$ is $\left(\varphi(\hat{x}), \lambda\left(\hat{x}^{*}\right)_{p}\right)$, where $\hat{x}:=(\lambda+\varphi)^{-1}(x)$. It follows that for all $\lambda>0$ we have

$$
\left.g_{\lambda}\right|_{S_{p}}=\left.g\right|_{S_{p}}, \quad g_{\lambda}\left(S_{p}, T_{p} N\right)=0 \text { and } g_{\lambda}\left(x^{*}, y^{*}\right)=g_{0}\left(\lambda \varphi(\lambda+\varphi)^{-1}(x), y\right)
$$

for all $x, y \in \mathfrak{m}$. From this, the first and the fourth assertion on the limits of $g_{\lambda}$ as $\lambda \rightarrow \infty$ and $\lambda \rightarrow 0$ follow. The second statement follows since

$$
\mu\left(\lambda \varphi(\lambda+\varphi)^{-1}\right)\left(\mu+\left(\lambda \varphi(\lambda+\varphi)^{-1}\right)\right)^{-1}=\frac{\mu \lambda}{\mu+\lambda} \varphi\left(\frac{\mu \lambda}{\mu+\lambda}+\varphi\right)^{-1},
$$

and the third follows since for $\lambda<\lambda_{0}$ we can write $\lambda=\frac{\mu \lambda_{0}}{\mu+\lambda_{0}}$ for $\mu:=\frac{\lambda \lambda_{0}}{\lambda_{0}-\lambda}>0$ and then apply the preceding statement and the fact that a Cheeger deformation of a nonnegatively curved metric is again nonnegatively curved.

\section{Homogeneous metrics of nonnegative curvature}

By virtue of Proposition 2.2, we should consider Cheeger deformations with small parameters $\lambda>0$ when searching for nonnegatively curved metrics. Moreover, since the $G$-orbits of $M$ are homogeneous spaces, the last statement of Proposition 2.2 implies that the metrics on the orbits approach a biinvariant metric for Cheeger deformations with small $\lambda>0$. Therefore, it is important to investigate the question which $G$-invariant metrics on a homogeneous space close to the normal homogeneous one have nonnegative curvature.

In general, this question is too hard to answer. But we can give a partial answer if $G / H$ is the total space of a homogeneous fibration. 
Theorem 3.1 ([STa1]) Let $H \subset K \subset G$ be compact Lie groups with Lie algebras $\mathfrak{h} \subset \mathfrak{k} \subset \mathfrak{g}$, and consider the homogeneous fibration $K / H \hookrightarrow G / H \rightarrow G / K$. Moreover, denote the orthogonal decompositions w.r.t. some biinvariant metric $g_{0}$ on $\mathfrak{g}$ as

$$
\mathfrak{g}=\mathfrak{h} \oplus \mathfrak{p}=\mathfrak{k} \oplus \mathfrak{s} \text { and } \mathfrak{k}=\mathfrak{h} \oplus \mathfrak{m}
$$

1. The metric on $G / H$ obtained from the normal homogeneous one by shrinking the fibers of this fibration is always nonnegatively curved.

2. The metric on $G / H$ obtained from the normal homogeneous one by enlarging the fibers of this fibration by a factor $(1+\varepsilon)$ is nonnegatively curved for $\varepsilon>0$ sufficiently small if and only if there is a constant $C>0$ such that for all $X, Y \in \mathfrak{p}$,

$$
\left|\left[X^{\mathfrak{m}}, Y^{\mathfrak{m}}\right]^{\mathfrak{m}}\right| \leq C \cdot|[X, Y]|,
$$

where the superscripts refer to the decomposition (1).

In particular, if the fiber $K / H$ is a symmetric space, then $\left[X^{\mathfrak{m}}, Y^{\mathfrak{m}}\right]^{\mathfrak{m}}=0$, so that in this case, the fiber can always be enlarged while maintaining nonnegative curvature. All metrics on $G / H$ in this theorem are induced by the left invariant metric given by the inner product

$$
g_{t}:=\left.t g_{0}\right|_{\mathfrak{k}}+\left.g_{0}\right|_{\mathfrak{s}}
$$

on $\mathfrak{g}$; indeed, shrinking (enlarging, respectively) the fibers corresponds to the case $t<1$ $(t>1$, respectively).

Proof. For the first statement, we observe that applying the Cheeger deformation to the action of $K$ on $G$ by right multiplication, we obtain that $g_{\lambda}$ is the left invariant metric which on $\mathfrak{g}=T_{e} G$ is given as

$$
g_{\lambda}=\left.\frac{\lambda}{1+\lambda} g_{0}\right|_{\mathfrak{k}}+\left.g_{0}\right|_{\mathfrak{s}},
$$

hence this metric on $G$ is nonnegatively curved, and so is the induced metric on $G / H$, which corresponds to scaling the fibers by $t:=\lambda /(1+\lambda) \in(0,1)$.

The second part follows from a more careful investigation of the curvature formula for invariant metrics on homogeneous spaces which we shall not present here. The details may be found in [STa1].

If $H$ is the trivial group so that $G / H=G$, then (2) in the above theorem is equivalent to a simpler criterion. Namely, we have the following

Theorem 3.2 ([S]) The left invariant metric on $G$ induced by (3) has nonnegative curvature for some $t>1$ if and only if the semi-simple part of $\mathfrak{k}$ is an ideal of $\mathfrak{g}$. In this case, this metric has nonnegative curvature for all $t \leq 4 / 3$. 


\section{Collar metrics of nonnegative curvature}

Let $M$ be a manifold with boundary $N:=\partial M$. A Riemannian metric $g$ on $M$ is called a collar metric, if there is a neighborhood of $N$ which is isometric to $\left([0, \varepsilon) \times N, d t^{2}+g_{N}\right)$ for some $\varepsilon>0$ and some metric $g_{N}$ on $N$. Evidently, if $M_{1}, M_{2}$ are manifolds with $\partial M_{1}=\partial M_{2}=: N$, then collar metrics $g_{i}$ on $M_{i}$ whose restriction to the boundary $N$ are isometric can be glued together by an isometry to a smooth metric on

$$
M:=M_{1} \cup_{N} M_{2} .
$$

This simple principle has proven very useful to construct metrics of nonnegative curvature, as the following examples due to J. Cheeger may illustrate.

Theorem 4.1 ([C]) Let $\hat{M}^{n}$ be a compact rank one symmetric space (CROSS), and let $M:=\hat{M}^{n} \backslash B_{\varepsilon}(p)$ be the complement of a small open ball. Then there is a nonnegatively curved collar metric on $M$ such that the metric on $\partial M=S^{n-1}$ is the round metric.

Corollary 4.2 ([C]) Let $M_{1}^{n}$ and $M_{2}^{n}$ be CROSSes of equal dimension. Then both $M_{1} \# M_{2}$ and $M_{1} \# \bar{M}_{2}$ admit nonnegatively curved metrics.

The corollary is an immediate consequence of the theorem, since the glueing in this case corresponds to taking the connected sum, and changing the orientation on one of the summands before glueing is possible. In order to prove the theorem, Cheeger used the fact that $M$ is always a homogeneous disk bundle over a CROSS of lower dimension and was able to perform the construction of the metric in a fairly explicit way.

If $M$ is a manifold with boundary $N=\partial M$ which admits a nonnegatively curved collar metric, then the Soul Theorem ([CG]) implies that there is a totally geodesic submanifold $S \subset M$, called the soul of $M$, such that $M$ is the total space of a unit disk bundle $D \rightarrow S$, whence $N \rightarrow S$ is a sphere bundle.

Given a disk bundle $M \rightarrow S$, it is thus a reasonable question to ask if $M$ admits a nonnegatively curved collar metric and if so, what are the possible restrictions of such a metric to $N=\partial M$. If $M \rightarrow S$ carries such a metric, then evidently it can be extended to a complete nonnegatively curved metric on the corresponding vector bundle $\hat{M} \rightarrow S$ by defining the metric to be the product $\left(\left[r_{0}, \infty\right) \times N, d t^{2}+g_{N}\right)$ on $\hat{M} \backslash M$. The converse of this statement also holds:

Theorem 4.3 ([G]) Let $(\hat{M}, g)$ be a complete open manifold of nonnegative curvature, so that by the soul theorem $\hat{M}$ is the total space of a vector bundle $\hat{M} \rightarrow S$. Then there is a nonnegatively curved collar metric on the unit disk bundle $M \subset \hat{M}$.

As an immediate consequence of this result, we may conclude the following.

Corollary 4.4 Let $\hat{M}_{k}, k=1,2$ be open manifolds admitting a complete nonnegatively curved metric, and let $M_{k} \subset \hat{M}_{k}$ be the corresponding unit disk bundles. Then there is a nonnegatively curved metric on the (closed) manifold

$$
\partial\left(M_{1} \times M_{2}\right)
$$


Proof. We may write $\partial\left(M_{1} \times M_{2}\right)=\left(M_{1} \times \partial M_{2}\right) \cup_{\partial M_{1} \times \partial M_{2}}\left(\partial M_{1} \times M_{2}\right)$. Now consider the nonnegatively curved collar metrics $g_{k}$ on $M_{k}$ whose existence is guaranteed by Theorem 4.3. Then the products of these metrics induce nonnegatively curved collar metrics on $M_{1} \times \partial M_{2}$ and $\partial M_{1} \times M_{2}$ whose boundary is isometric to $\left(\partial M_{1} \times \partial M_{2}, g_{1}+g_{2}\right)$, so that these metrics may be glued together.

\section{Bundles with normal homogeneous collar}

A special class of vector bundles which we wish to consider are homogeneous vector bundles and disk bundles. These are bundles of the form

$$
\hat{M}:=G \times_{K} V \text { and } M:=G \times_{K} \overline{B_{R}(0)} \subset G \times_{K} V \text {, respectively, }
$$

where $K \subset G$ acts on the Euclidean vector space $V$ by some orthogonal representation $K \rightarrow O(V)$ which is transitive on the unit sphere $S^{V} \subset V$, so that $S^{V}=K / H$ for some subgroup $H \subset K$.

Note that $\hat{M}$ is the quotient of $G \times V$ by the free left action of $K$ given by $k \star(g, v):=$ $\left(g k^{-1}, k \cdot v\right)$. Thus, any $K$-invariant nonnegatively curved metric on $G \times V$ induces a nonnegatively curved metric on $\hat{M}$, and this metric on $M \subset \hat{M}$ is a collar metric if the metric on $G \times \overline{B_{R}(0)} \subset G \times V$ is.

If follows that each homogeneous disk bundle has $G$-invariant nonnegatively curved collar metrics: choose on $G \times \overline{B_{R}(0)}$ the direct sum of a nonnegatively curved left invariant, $A d_{K^{-}}$ invariant metric on $G$ and a nonnegatively curved $K$-invariant collar metric on $\overline{B_{R}(0)}$, such as a "cigar metric", i.e., a nonnegatively curved $O(V)$-invariant metric which is a cylinder outside a compact set. Clearly, such metrics exist. However, the induced metric on the boundary $G / H$ is in general not normal homogeneous, and this limits the possibilities of glueing two such bundles.

Definition 5.1 Let $M:=G \times_{K} \overline{B_{R}(0)} \subset G \times_{K} V \longrightarrow G / K$ be a homogeneous disk bundle with $K \rightarrow O(V)$ acting transitively on the sphere $S^{V}=K / H$. A metric on $M$ is called a normal homogeneous collar metric if a neighborhood of the boundary $\partial M \cong G / H$ is $G$ equivariantly isometric to $\left([0, \varepsilon) \times G / H, d t^{2}+g_{0}\right)$, where $g_{0}$ is a normal homogeneous metric on $G / H$.

Let us suppose that the metric on $M$ is a submersion of $\left(G \times \overline{B_{R}(0)}, g_{\mathfrak{g}}+g_{V}\right)$ where $g_{\mathfrak{g}}$ is a left invariant $A d_{K}$-invariant metric on $G$ and $g_{V}$ is a $K$-invariant collar metric on $\overline{B_{R}(0)}$ whose boundary metric on $K / H=S^{V} \subset V$ is induced by a left invariant metric $\mathfrak{g}_{\mathfrak{k}}$ on $K$. Then the metric on the boundary $\partial M \cong G / H$ is induced by the Riemannian submersion

$$
\left(G \times K, g_{\mathfrak{g}}+g_{\mathfrak{k}}\right) \longrightarrow G \longrightarrow G / H .
$$

It is now of interest to see how these metrics on $G$ and $\overline{B_{R}(0)} \subset V$ can be chosen such that the submersion (4) induces a normal homogeneous metric on $G / H$ and hence a normal 
homogeneous collar metric on $M$. For the metric on $\overline{B_{R}(0)} \subset V$, we may use the following result.

Theorem 5.2 ([STu]) Let $K \rightarrow O(V)$ be a representation of a Lie group $K$ on a Euclidean vector space $V$ which is transitive on the unit sphere $S^{V} \subset V$. Then there is a nonnegatively curved collar metric on $\overline{B_{R}(0)} \subset V$ which is $N_{\text {orm }}(V) K$-invariant and is normal homogeneous on $S^{V}=K / H$.

Let $L \rightarrow O(V)$ be a representation which is transitive on the unit sphere $S^{V} \subset V$, and extend this to a representation $K \times L \rightarrow O(V)$, i.e., $K$ maps to the centralizer $Z_{O(V)}(L)$. Since the normal homomogeneous collar metric on $V$ from the Theorem 5.2 is $(K \times L)$ invariant, there is an induced nonnegatively curved normal homogeneous collar metric on

$$
(G \times L) \times_{K \times L} V \longrightarrow G / K,
$$

which as a bundle can be regarded as $G \times_{K} V \rightarrow G / K$, where $K$ acts on $V$ as a subgroup of $Z_{O(V)}(L) \subset O(V)$. Such a homogeneous bundle is called essentially trivial. Thus, all essentially trivial homogeneous disk bundles carry a nonnegatively curved collar metric whose boundary is normal homogeneous w.r.t. the action of $G \times L$.

The following gives an exhaustive description of all essentially trivial bundles which are not trivial.

Example 5.3 1. $\mathbb{Z}_{2}$-quotients. Let $K \triangleleft K^{\prime} \subset G$ be such that $K^{\prime} / K \cong \mathbb{Z}_{2}$, and let $L \rightarrow O(V)$ be a representation which is transitive on the unit sphere. Moreover, define the representation $K^{\prime} \rightarrow K^{\prime} / K \cong\{ \pm I d\} \subset O(V)$. Then the essentially trivial bundle

$$
(G \times L) \times_{K^{\prime} \times L} V \cong G \times_{K^{\prime}} V \longrightarrow G / K^{\prime},
$$

is the quotient of the trivial bundle $G / K \times V$ by $\mathbb{Z}_{2} \cong K^{\prime} / K$ which acts on $G / K$ from the right and on $V$ as $\{ \pm I d\}$.

2. Rank one bundles. If $\operatorname{dim} V=1$, i.e., $V=\mathbb{R}$, then $O(V) \cong \mathbb{Z}_{2}$, hence any homogeneous bundle of rank one is of the form $G \times_{K^{\prime}} V \rightarrow G / K^{\prime}$ with a surjective map $K^{\prime} \rightarrow O(V) \cong \mathbb{Z}_{2}$. The kernel of this map is a normal subgroup $K \triangleleft K^{\prime}$ with $K^{\prime} / K \cong \mathbb{Z}_{2}$. Thus, this is a special case of the preceding, i.e., we can write any non-trivial rank one homogeneous bundle in the form

$$
\left(G \times \mathbb{Z}_{2}\right) \times_{K^{\prime} \times \mathbb{Z}_{2}} V \cong G \times_{K^{\prime}} V \rightarrow G / K^{\prime},
$$

which is the quotient $(G / K \times V) / \mathbb{Z}_{2}$ as above.

3. $S^{1}$-quotients. Similarly, if $V$ is a complex hermitean vector space and $L \rightarrow U(V)$ is transitive on the unit sphere, then we consider subgroups $K \triangleleft K^{\prime} \subset G$ such that $K^{\prime} / K=S^{1}$. Then the essentially trivial bundle

$$
(G \times L) \times_{K^{\prime} \times L} V \cong G \times{ }_{K^{\prime}} V \longrightarrow G / K^{\prime},
$$

is the quotient of the trivial bundle $G / K \times V$ by the action of $S^{1}=K^{\prime} / K$ on $G / K$ from the right and on $V$ by scalar multiplication by $S^{1} \subset \mathbb{C}$. 
4. Sp(1)-quotients. Likewise, let $V$ be a quaternionic hermitean vector space and suppose that $L \rightarrow S p(V)$ is surjective and hence transitive on the unit sphere. Again, consider subgroups $K \triangleleft K^{\prime} \subset G$ such that $K^{\prime} / K=S p(1)$. Then the essentially trivial bundle

$$
(G \times L) \times_{K^{\prime} \times L} V \cong G \times_{K^{\prime}} V \longrightarrow G / K^{\prime}
$$

is the quotient of the trivial bundle $G / K \times V$ by the action of $S p(1)=K^{\prime} / K$ on $G / K$ from the right and on $V$ by scalar multiplication by $S p(1) \subset \mathbb{H}$.

Thus, all these bundles admit nonnegatively curved normal homogeneous collar metrics. Note that this description of essentially trivial bundles is exhaustive, since by the classification of transitive actions of a Lie group $L$ on a sphere $S^{V} \subset V$ the centralizer $Z_{O(V)}(L) \subset O(V)$ is either trivial or one of $\mathbb{Z}_{2}, S^{1}$ or $S p(1)$.

By virtue of Theorem 5.2, we may assume that $g_{\mathfrak{k}}$ in (4) is a biinvariant metric. If we choose $g_{\mathfrak{g}}$ as a biinvariant metric such that $g_{\mathfrak{k}}=\left.\left(g_{\mathfrak{g}}\right)\right|_{\mathfrak{k}}$, then it follows that the induced metric on $G / H$ is obtained by shrinking the fiber of the normal homogeneous metric of the fibration $S^{V}=K / H \hookrightarrow G / H \rightarrow G / K$. In particular, this metric on the boundary $G / H$ is not normal homogeneous.

In order to achieve a normal homogeneous boundary metric on $G / H$, we have to define the metric on $\mathfrak{g}=\mathfrak{k} \oplus \mathfrak{s}$ by

$$
g_{\mathfrak{g}}:=\left.(1+\varepsilon)\left(g_{0}\right)\right|_{\mathfrak{k}}+\left.\left(g_{0}\right)\right|_{\mathfrak{s}},
$$

where $g_{0}$ is a biinvariant metric. When choosing $g_{\mathfrak{k}}:=\left.\left(1+\varepsilon^{-1}\right)\left(g_{0}\right)\right|_{\mathfrak{k}}$, then (4) induces the metric $g_{0}$ on $G$ and hence a normal homogeneous metric on $G / H$.

Recall from Theorem 3.2 that the metric $g_{\mathfrak{g}}$ from (5) has nonnegative curvature for some $\varepsilon>0$ if and only if the semi-simple part of $\mathfrak{k}$ is an ideal of $\mathfrak{g}$. In particular, this is satisfied if $\operatorname{dim}(V) \leq 2$ in which case $\mathfrak{k}$ is at most one-dimensional and hence abelian. Therefore, we obtain the following result.

Theorem 5.4 ([GZ]) Let $M=G \times_{K} V$ be a homogeneous disk bundle of rank $\operatorname{dim} V \leq 2$. Then $M$ admits a nonnegatively curved normal homogeneous collar metric.

However, if $\operatorname{dim} V \geq 3$ and the bundle $M \rightarrow G / K$ is not essentially trivial, then the semi-simple part of $\mathfrak{k}$ is not an ideal. Thus, by Theorem 3.2, the left invariant metric $g_{\mathfrak{g}}$ from (5) has some negative curvature on $G$. But in order for the submersion metric on $M=G \times \times_{K} V$ to be nonnegatively curved, it suffices that $g_{\mathfrak{g}}$ has nonnegative curvature on all planes which are contained in $\mathfrak{p}$. Some interesting examples where this occurs have been described in [STa1]. Once again, the proof of this theorem involves a direct calculation of the curvature formula for homogeneous metrics.

Theorem 5.5 ([STa1]) Let $H \subset K \subset G$ be compact Lie groups with Lie algebras $\mathfrak{h} \subset \mathfrak{k} \subset \mathfrak{g}$, and consider the decompositions from (1). If there exists $C>0$ such that for all $X=$ $X^{\mathfrak{m}}+X^{\mathfrak{s}}, Y=Y^{\mathfrak{m}}+Y^{\mathfrak{s}} \in \mathfrak{m} \oplus \mathfrak{s}=\mathfrak{p}$ we have

$$
\left|X^{\mathfrak{m}} \wedge Y^{\mathfrak{m}}\right| \leq C \cdot|[X, Y]|
$$


then any left invariant metric on $G$ sufficiently close to $g_{0}$ which is $A d_{H}$-invariant and is a constant multiple of $g_{0}$ on $\mathfrak{s}$ and $\mathfrak{h}$ (but arbitrary on $\mathfrak{m}$ ) has nonnegative sectional curvature on all planes contained in $\mathfrak{p}$.

That is, for all Lie groups $H \subset K \subset G$ satisfying this criterion, the metric $g_{\mathfrak{g}}$ from (5) has nonnegative curvature on all planes contained in $\mathfrak{p}$ for small $\varepsilon>0$, hence if $K / H=S^{V}$, then the corresponding homogeneous vector bundle $G \times_{K} V \rightarrow G / K$ has a nonnegatively curved normal homogeneous collar metric.

As it turns out, condition (6) is almost necessary and sufficient for a homogeneous disk bundle to admit such a metric. Namely, we have

Theorem 5.6 ([STa2]) Let $G \times{ }_{K} V \rightarrow G / K$ be a homogeneous vector bundle which admits a nonnegatively curved normal homogeneous collar metric, let $H \subset K$ be the subgroup for which $K / H=S^{V}$, and decompose the Lie algebras $\mathfrak{h} \subset \mathfrak{k} \subset \mathfrak{g}$ as in (1).

If $\mathfrak{m}_{1} \subset \mathfrak{m}$ is any non-trivial Ad${ }_{H}$-irreducible subspace such that $\mathfrak{m}$ contains no other $A d_{H}$-irreducible factor equivalent to $\mathfrak{m}_{1}$, then there exists a constant $C>0$ such that for all $X=X^{\mathfrak{m}}+X^{\mathfrak{s}}, Y=Y^{\mathfrak{m}}+Y^{\mathfrak{s}} \in \mathfrak{m}_{1} \oplus \mathfrak{s}$ condition (6) holds.

When $K / H$ is isotropy irreducible, the choice $\mathfrak{m}_{1}=\mathfrak{m}$ yields a converse to Theorem 5.5. The proof of this theorem uses Perelman's result on the existence of parallel vector fields on a totally convex set $([\mathrm{P}])$. This implies that along a normal geodesic $c(t)$ the metric on the principal orbit through $c(t)$ is induced by a metric of the form $g_{t}=\left.\left(g_{0}\right)\right|_{\mathfrak{s}}+\left.\tilde{g}_{t}\right|_{\mathfrak{k}}$. Then one shows that such a metric must have some negative curvature close to the collar unless (6) is satisfied.

With these two results, one can give an almost complete classification of homogeneous disk bundles which admit nonnegatively curved normal homogeneous collar metrics.

Theorem 5.7 ([STa2]) Let $M:=G \times_{K} V \rightarrow G / K$ be a cohomogeneity one homogeneous vector bundle which is essentially non-trivial. If $M$ admits a $G$-invariant normal homogeneous collar metric of nonnegative curvature, then the rank of this bundle must be in $\{2,3,4,6,8\}$.

By Theorem 5.4, all rank two bundles admit a $G$-invariant normal homogeneous collar metric of nonnegative curvature. In the higher rank case, the situation is much more restricted. For rank eight, we have the following complete classification.

Theorem 5.8 ([STa2]) Let $M:=G \times_{K} V \rightarrow G / K$ be a G-irreducible cohomogeneity one homogeneous vector bundle which is essentially non-trivial and such that $\operatorname{dim}_{\mathbb{R}} V=8$. Then $M$ admits a $G$-invariant normal homogeneous collar metric of nonnegative curvature if and only if $M$ is finitely $G$-equivariantly covered by one of the following:

1. $\operatorname{Spin}(p+9) \times_{\operatorname{Spin}(8)} \mathbb{R}^{8}$ for $p \in\{0,1,2\}$, where $\operatorname{Spin}(8)$ acts on $\mathbb{R}^{8}$ by a spin representation, and $\operatorname{Spin}(8) \subset \operatorname{Spin}(p+9)$ is the lift of the standard inclusion $S O(8) \subset S O(p+9)$. 
2. $\operatorname{Spin}(p+8) \times_{\operatorname{Spin}(7)} \mathbb{R}^{8}$ for $p \in\{0,1\}$, where $\operatorname{Spin}(7)$ acts on $\mathbb{R}^{8}$ by the spin representation, and $\operatorname{Spin}(7) \subset \operatorname{Spin}(p+8)$ is the lift of the standard inclusion $S O(7) \subset S O(p+8)$.

3. $\operatorname{Spin}(7) \times{ }_{\operatorname{Spin}(6)} \mathbb{C}^{4}$, with the standard representation of $\operatorname{Spin}(6) \cong S U(4)$ on $\mathbb{C}^{4}$.

4. A quotient of one of the preceding examples:

(a) $\left(\operatorname{Spin}(p+9) \cdot G^{\prime}\right) \times_{\operatorname{Spin}(8) \cdot H^{\prime}} \mathbb{R}^{8}$ for $p \in\{1,2\}$ and an arbitrary compact Lie group $G^{\prime}$ and $H^{\prime} \subset \operatorname{Spin}(p+1) \cdot G^{\prime}$ with $H^{\prime} \not \subset G^{\prime}$, which acts trivially on $\mathbb{R}^{8}$.

(b) $\left(\operatorname{Spin}(9) \cdot G^{\prime}\right) \times_{\operatorname{Spin}(7) \cdot H^{\prime}} \mathbb{R}^{8}$ for an arbitrary compact Lie group $G^{\prime}$ and $H^{\prime} \subset$ $\operatorname{Spin}(2) \cdot G^{\prime}=S^{1} \cdot G^{\prime}$ with $H^{\prime} \not \subset G^{\prime}$, which acts trivially on $\mathbb{R}^{8}$.

(c) $\left(\operatorname{Spin}(7) \cdot G^{\prime}\right) \times_{S p i n(6) \cdot S^{1} \cdot H^{\prime}} \mathbb{C}^{4}$ for an arbitrary compact Lie group $G^{\prime} \supset S^{1} \cdot H^{\prime}$, where $S^{1} \subset G^{\prime}$ acts on $\mathbb{C}^{4}$ by multiples of the identity, and $H^{\prime} \subset G^{\prime}$ acts trivially.

Here, for Lie groups $L_{1}, L_{2}$, we denote by $L_{1} \cdot L_{2}$ the quotient of $L_{1} \times L_{2}$ by a finite subgroup of the center. The term $G$-irreducible means that $M$ is not $G$-equivariantly finitely covered by a bundle of the form $\left(G_{1} / H_{1}\right) \times M^{\prime}$ with $\operatorname{dim}\left(G_{1} / H_{1}\right)>0$ and $M^{\prime}$ a cohomogeneity one homogeneous vector bundle. This hypothesis is natural because, for $G$-reducible bundles, our problem easily reduces to deciding whether $M^{\prime}$ admits such a metric.

For rank 6 bundles, we obtain the following

Theorem 5.9 ([STa2]) Let $M:=G \times_{K} V \rightarrow G / K$ be a G-irreducible cohomogeneity one homogeneous vector bundle which is essentially non-trivial and such that $\operatorname{dim}_{\mathbb{R}} V=6$. Then $M$ admits a $G$-invariant normal homogeneous collar metric of nonnegative curvature if and only if $M$ is finitely $G$-equivariantly covered by one of the following:

1. $S U(5) \times_{S U(4)} \mathbb{R}^{6}$, with the irreducible action of $S U(4) \cong \operatorname{Spin}(6)$ on $\mathbb{R}^{6}$.

2. $\left(S U(5) \cdot G^{\prime}\right) \times_{S U(4) \cdot H^{\prime}} \mathbb{R}^{6}$ for an arbitrary compact Lie group $G^{\prime}$ and $H^{\prime} \subset S^{1} \cdot G^{\prime}$ with $S^{1} \subset S U(5)$ being the centralizer of $S U(4)$, and $H^{\prime} \not \subset G^{\prime}$ acts trivially on $\mathbb{R}^{6}$.

To describe our results for rank three and four bundles, we require some notation for subgroups of the exceptional Lie group $G_{2}$. Let $S O(4) \subset G_{2}$ and $S U(3) \subset G_{2}$ be the isotropy groups of the symmetric space $G_{2} / S O(4)$ and the sphere $S^{6}=G_{2} / S U(3)$, respectively. After conjugating these groups appropriately, their intersection can be made isomorphic to $U(2)$, and we let $S U(2)_{1} \subset S O(4) \cap S U(3) \subset G_{2}$ be the simple part of this intersection. Note that $S U(2)_{1} \subset S O(4)$ is normal, and we denote its centralizer in $G_{2}$ by $S U(2)_{3} \subset S O(4) \subset$ $G_{2}$. (The subscripts of the $S U(2)$-subgroups of $S O(4)$ denote their maximal weight for the isotropy representation of $G_{2} / S O(4)$.) Using this notation, we can make the following statement about the rank three case.

Theorem 5.10 ([STa2]) Let $M:=G \times_{K} V$ be a G-irreducible cohomogeneity one homogeneous vector bundle which is essentially non-trivial such that $\operatorname{dim}_{\mathbb{R}} V=3$. If $M$ admits a nonnegatively curved $G$-invariant normal homogeneous collar metric, then $M$ must be finitely $G$-equivariantly covered by one of the following. 
1. $M_{1}=G_{2} \times_{S O(4)} \mathfrak{s u}(2)_{3}$, where $S O(4)$ acts on $\mathfrak{s u}(2)_{3} \triangleleft \mathfrak{s o}(4)$ by the adjoint representation.

2. $M_{2}=\left(S p(p+1) \cdot G^{\prime}\right) \times_{S p(1) \cdot H^{\prime}} \mathfrak{s p}(1)$ with $H^{\prime} \subset S p(p) \cdot G^{\prime}$, where $S p(1) \cdot H^{\prime}$ acts on $\mathfrak{s p}(1) \triangleleft \mathfrak{s p}(1) \oplus \mathfrak{h}^{\prime}$ by the adjoint representation.

Further, $M_{1}$ admits such a metric, as does $M_{2}$ with $G^{\prime}=1$ and $H^{\prime}=S p(p)$.

Finally, in the rank four case, we have the following examples, which are all related to those in Theorem 5.10 .

Theorem 5.11 ([STa2]) The following cohomogeneity one homogeneous vector bundles (orbifold bundles, respectively) of rank four admit $G$-invariant normal homogeneous collar metrics of nonnegative curvature:

1. $G_{2} \times_{S O(4)}(\mathbb{H} / \pm 1)$, where $S U(2)_{1} \subset S O(4)$ acts trivially and $S U(2)_{3} \subset S O(4)$ by left multiplication on $\mathbb{H} / \pm 1$. Note that this is an orbifold bundle only.

2. $\left(G_{2} \times G^{\prime}\right) \times_{S O(4) \times S U(2)^{\prime}}(\mathbb{H} / \pm 1)$, where $S U(2)_{1} \subset S O(4)$ acts trivially and $S U(2)_{3} \subset$ $S O(4)$ by left multiplication, whereas $S U(2)^{\prime} \subset G^{\prime}$ acts by right multiplication on $\mathbb{H} / \pm 1$ with $G^{\prime}$ arbitrary. Note that these are orbifold bundles only.

3. $S p(p+1) \times_{S p(1) \times S p(p)} \mathbb{H}$ where $S p(p)$ acts trivially and $S p(1)$ by left multiplication on $\mathbb{H}$.

4. $\left(S p(p+1) \times G^{\prime}\right) \times_{S p(1) \times S p(p) \times S p(1)^{\prime}} \mathbb{H}$ where $S p(p)$ acts trivially and $S p(1)$ by left multiplication, whereas $S p(1)^{\prime} \subset G^{\prime}$ acts by right multiplication on $\mathbb{H}$ with $G^{\prime}$ arbitrary.

\section{Cohomogeneity one manifolds}

A cohomogeneity one manifold is a manifold $M$ with an action of a compact Lie group $G$ such that $\operatorname{dim}(M / G)=1$. For a cohomogeneity one manifold, there are four possibilities for the quotient space.

1. $M / G \cong \mathbb{R}$. In this case, $M$ is equivariantly diffeomorphic to $\mathbb{R} \times G / H$ for some subgroup $H \subset G$. In particular, $M$ admits a $G$-invariant metric of nonnegative curvature.

2. $M / G \cong[0, \infty)$. In this case, $M$ has one non-principal orbit and is a homogeneous vector bundle as described in the preceding section. In particular, $M$ admits a $G$-invariant metric of nonnegative curvature.

3. $M / G \cong S^{1}$. Then $M$ is finitely covered by $S^{1} \times G / H$ for some subgroup $H \subset G$. Again, $M$ admits a $G$-invariant metric of nonnegative curvature. 
4. $M / G \cong[-1,1]$. In this case, $M$ has two non-principal orbits and can be written as

$$
M=D_{-} \cup_{G / H} D_{+}, \text {where } D_{ \pm}=G \times_{K_{ \pm}} V_{ \pm}
$$

are homogeneous disk bundles with common boundary $G / H$. That is, $H \subset K_{ \pm}$and $K_{ \pm} / H$ is the unit sphere $S^{V_{ \pm}} \subset V_{ \pm}$.

It is the last of these classes which we are interested in, as it is obtained by glueing two homogeneous disk bundles together along their common boundary which is a principal orbit. Conversely, given Lie groups $H \subset\left\{K_{+}, K_{-}\right\} \subset G$ such that $K_{ \pm} / H$ is a sphere, then there is a unique cohomogeneity one manifold which is determined by these groups. Thus, these groups can be used to identify the manifold.

As it turns out, not all cohomogeneity one manifolds with two singular orbits admit invariant metrics of nonnegative curvature ([GVWZ]). However, we have the following result.

Theorem 6.1 ([STu]) Let $M$ be a closed cohomogeneity one manifold. Then $M$ admits $G$ invariant metrics of almost nonnegative curvature, i.e., there is a sequence of $G$-invariant metrics $g_{n}$ on $M$ such that $\operatorname{diam}\left(M, g_{n}\right)^{2} \sec \left(M, g_{\mathfrak{n}}\right)>-1 / n$.

This is proven once again using Cheeger deformations: Let $g_{M}$ be any $G$-invariant metric on $M$, and let $g_{0}$ be a biinvariant metric on $G$. Then the metric $g_{\lambda}$ on $M$ induced by the submersion $\left(G \times M, \lambda g_{0}+g_{M}\right) \rightarrow\left(M, g_{\lambda}\right)$ has uniformly bounded diameter for small $\lambda>0$, and one verifies that the lower curvature bound of $g_{\lambda}$ tends to 0 as $\lambda \rightarrow 0$.

From the results in the preceding section, we see that we obtain a nonnegatively curved metric on a cohomogeneity one manifold with two singular orbits if the corresponding two disk bundles admits nonnegatively curved normal homogeneous collar metrics. Thus, Theorem 5.4 immediately implies

Theorem 6.2 ([GZ]) Let $M$ be a cohomogeneity one manifold with two non-principal orbits of codimension at most 2 . Then $M$ admits a $G$-invariant metric of nonnegative curvature.

This result yields some spectacular examples of manifolds which admit nonnegatively curved metrics, such as all four (unoriented) diffeomorphism types of $\mathbb{R} \mathbb{P}^{5}$ and ten out of the fourteen (unoriented) exotic spheres of dimension 7.

Unfortunately, the remaining homogeneous disk bundles admitting nonnegatively curved normal homogeneous collar metrics do not yield new examples of nonnegatively curved cohomogeneity one manifolds, since glueing two of them either is the restriction of a homogeneous action on $M$, or there is a Riemannian submersion $\hat{M} \rightarrow M$, where $\hat{M}$ is known to have a nonnegatively curved metric.

For instance, glueing together two disk bundles of the second type of Theorem 5.8 with $p=0$, after applying outer automorphisms of $\operatorname{Spin}(8)$, we conclude that the primitive cohomogeneity one manifold given by the group diagram $G_{2} \subset\left\{\operatorname{Spin}_{+}(7), \operatorname{Spin}_{-}(7)\right\} \subset \operatorname{Spin}(8)$ admits a metric of nonnegative curvature with a totally geodesic normal homogeneous principal orbit. However, this manifold is diffeomorphic to the sphere $S^{15}$ ([GWZ]). 


\section{References}

[C] J. Cheeger, Some examples of manifolds of nonnegative curvature, J. Diff. Geom. 8 (1973) $623-628$

[CG] J. Cheeger, D. Gromoll, On the structure of complete manifolds of nonnegative curvature, Ann. of Math. 96 (1972) 413-43.

[GVWZ] K. Grove, L. Verdiani, B. Wilking, W. Ziller, Nonnegative curvature obstructions in cohomogeneity one and the Kervaire spheres, Ann. Sc. Norm. Super. Pisa, Cl. Sci. (5) 5, No. 2 (2006) 159-170

[GWZ] K. Grove, B. Wilking, W. Ziller, Positively curved cohomogeneity one manifolds and 3Sasakian geometry, J. Diff. Geom. 78 (2008) 33-111.

[GZ] K. Grove, W. Ziller, Curvature and symmetry of Milnor spheres, Ann. of Math. (2) 152 (2000) $331-36$

[G] L. Guijarro, Improving the metric in an open manifold with nonnegative curvature, Proc. AMS, 126 (1998) 1541-1545

[P] G. Perelman, Proof of the soul conjecture of Cheeger and Gromoll, J. Diff. Geom. 40 (1994), 209-212.

[S] L. Schwachhöfer, A remark on left invariant metrics on compact Lie groups, Arch.Math. 90 (2008) $158-162$

[STa1] L. Schwachhöfer, K. Tapp, Homogeneous Metrics with nonnegative curvature, Jour. Geom. Anal. 19 (2009) 929-943

[STa2] L. Schwachhöfer, K. Tapp, Cohomogeneity one disk bundles with normal homogeneous collars, Proc. London Math. Soc. 99, No.3 (2009) 609-632

[STu] L. Schwachhöfer and W. Tuschmann, Almost nonnegative curvature and cohomogeneity one, Preprint no. 62/2001, Max-Planck-Institut für Mathematik in den Naturwissenschaften Leipzig, http://www.mis.mpg.de/cgi-bin/preprints.pl

[Z] W. Ziller, Examples of Riemannian manifolds with nonnegative sectional curvature, Metric and Comparison Geometry, Surv. Diff. Geom. 11, ed. K.Grove and J.Cheeger, Intern. Press, 2007

Fakultät für Mathematik, Technische Universität Dortmund, Vogelpothsweg 87, 44221 Dortmund, Germany

E-mail address: lschwach@math.uni-dortmund.de 


\section{Preprints ab 2008/10}

2009-16

2009-15

2009-14

2009-13

2009-12

2009-11

2009-10

2009-09

2009-08

2009-07

2009-06

2009-05

2009-04

2009-03

2009-02

2009-01
Lorenz J. Schwachhöfer

Nonnegative curvature on disk bundles

\section{Iuliu Pop and Ben Schweizer}

Regularization schemes for degenerate Richards equations and outflow conditions

Guy Bouchitté and Ben Schweizer

Cloaking of small objects by anomalous localized resonance

\section{Tom Krantz, Lorenz J. Schwachhöfer}

Extrinsically Immersed Symplectic Symmetric Spaces

\section{Alexander Kaplun}

Continuous time Ehrenfest process in term structure modelling

Henryk Zähle

Ein aktuarielles Modell für die Portabilität der Alterungsrückstellungen in der PKV

Andreas Neuenkirch and Henryk Zähle

Asymptotic error distribution of the Euler method for SDEs with non-Lipschitz coefficients

Karl Friedrich Siburg, Pavel A. Stoimenov

Regression dependence

\section{Wilfried Hazod}

Continuous convolution hemigroups integrating a sub-multiplicative function

\section{Sergio Conti and Ben Schweizer}

On optimal metrics preventing mass transfer

\section{Simon Castle, Norbert Peyerimhoff, Karl Friedrich Siburg}

Billiards in ideal hyperbolic polygons

\section{Ludwig Danzer}

Quasiperiodic Tilings - Substitution Versus Inflation

\section{Flavius Guiaş}

Direct simulation of the infinitesimal dynamics of semi-discrete approximations for convection-diffusion-reaction problems

\section{Franz Kalhoff and Victor Pambuccian}

Existential definability of parallelism in terms of betweenness in Archimedean ordered affine geometry

\section{Fulvia Buzzi, Michael Lenzinger and Ben Schweizer}

Interface conditions for degenerate two-phase flow equations in one space dimension

\section{Henryk Zähle}

Approximation of SDEs by population-size-dependent Galton-Watson processes 
Mehler semigroups, Ornstein-Uhlenbeck processes and background driving Lévy processes on locally compact groups and on hypergroups

$2008-24$

$2008-23$

$2008-22$

$2008-21$

$2008-20$

2008-19

$2008-18$

$2008-17$

2008-16

$2008-15$

$2008-14$

2008-13

$2008-12$

2008-11
Karl Friedrich Siburg, Pavel A. Stoimenov

Symmetry of functions and exchangeability of random variables

\section{Ina Kirsten Voigt}

Voronoi Cells of Discrete Point Sets

Michael Lenzinger and Ben Schweizer

Effective reaction rates of a thin catalyst layer

\section{Michael Voit}

Bessel convolutions on matrix cones: Algebraic properties and random walks

\section{Margit Rösler and Michael Voit}

Limit theorems for radial random walks on $p \times q$-matrices as $p$ tends to infinity

\section{Michael Voit}

Central Limit Theorems for Radial Random Walks on $p \times q$ Matrices for $p \rightarrow \infty$

\section{Michael Voit}

Limit theorems for radial random walks on homogeneous spaces with growing dimensions

\section{Ansgar Steland and Henryk Zähle}

Sampling inspection by variables: nonparametric setting

\section{Guy Bouchitté and Ben Schweizer}

Homogenization of Maxwell's equations with split rings

\section{Wilfried Hazod}

Multiple selfdecomposable laws on vector spaces and on groups:

The existence of background driving processes

\section{Wilfried Hazod}

Mixing of generating functionals and applications to (semi-)stability of probabilities on groups

\section{Wilfried Hazod}

Probability on Matrix-Cone Hypergroups: Limit Theorems and Structural Properties

\section{Michael Lenzinger and Ben Schweizer}

Two-phase flow equations with outflow boundary conditions in the hydrophobic-hydrophilic case

\section{Karl Friedrich Siburg}

Geometric proofs of the two-dimensional Borsuk-Ulam theorem 